\title{
Stochastic Modeling and Performance Measures of Redundant System Operating in Different Conditions
}

\author{
Ibrahim Yusuf \\ Department of Mathematical Sciences, \\ Bayero University, Kano, Nigeria
}

\author{
Saminu I. Bala \\ Department of Mathematical Sciences, \\ Bayero University, Kano, Nigeria
}

\begin{abstract}
Many authors have studied repairable redundant system operating in different condition under the assumption that the system received corrective maintenance at failure and preventive maintenance before failure. However, little or no attention is paid on whether the system can receive minor and major maintenance when it is operating in a reduced capacity state. In this study we consider a redundant air condition cooling system consisting of main unit and a warm standby reserved unit operating in different weather condition (High and low temperature). Using Kolmogorov's forward equations method, various measures of system performance such as Mean time to system failure (MTSF), availability, busy period and frequency of preventive, minor and major maintenance, profit function are obtained. Mean time to system failure, availability and profit function are studied graphically.
\end{abstract}

\section{Keywords}

Profit analysis, MTSF, minor maintenance, major maintenance, preventive maintenance, availability.

\section{INTRODUCTION}

Provision of standby unit is vital towards achieving high reliability. System Reliability is improved through a standby unit support which is capable of performing similar function with the operational unit but with different degree and desirability. Redundancy is a technique used to improve system reliability and availability. It consists of techniques for increasing system effectiveness through reducing failure and maintenance cost minimization.

A large volume of literature exists in the area of reliability theory of standby systems which deals with models of obtaining reliability measures of the system effectiveness such as the mean time to system failure (MTSF), availability, busy period, profit function, expected frequency of replacement, etc. Frenkel et al [4] deal with the study of an aging air condition system operating at varing temperature condition with minimal repair at failure. The system consist of main and reserved unit, where the main air conditioner unit has levels: perfect function, reduced capacity, complete failure due to filter obstruction, and complete failure due to another reason. Using Markov reward model for non homogeneous poisson process, an optimal maintenance contract that maximizes the expected cost is obtained. El Demcese and Temraz [3] study an air condition system used in hospital comprising of three identical air conditioners in parallel with level of operation as : peak, middle, and low. Two situations were considered which are: (a) air conditioners failure and repair rates are time varying and (b) air conditioners failure and repair rates are constant. Using homogeneous and non homogeneous Markov reward model, models for computation of availability and reliability measures were developed.

El Said [1] deals with the cost analysis of two unit cold standby redundant system with two types of failures and preventive maintenance using Kolmogorov's forward equations, Haggag [5] discussed the cost analysis of analysis of a two unit cold standby redundant system under two types of failures involving common cause failures and preventive maintenance using kolmogorov's forward equation method, El -Sherbeny [2] performed comparative analysis of availability between two different system with general repairs, two types of failures and standby switch. Kumar and Malik [6] deals with stochastic modeling of two identical units computer system with priority to preventive maintenance over software replacement. Wang et al [7] performed comparative analysis of availability between two system with warm standby units and different imperfect coverage. Wang et al [8] studied cost benefit analysis of series system with cold standby components and a repairable service station.

In the present paper, we consider a two non identical unit air condition cooling system operating in high and low temperature condition. The system is attended by only one repairman.. The main unit can be in: operation with full capacity, operation with reduced capacity and failure, while the reserve unit can be in: operation with full capacity and failure. While in reduced capacity states, the main unit received minor or major maintenance depending upon its strength in reduced capacity states. The system is analyzed using kolmogorov's forward equations method and expressions for some reliability characteristics such as MTSF, availability, busy period, frequency of preventive, minor and major maintenance, profit function are derived. Numerical results are obtained to depicts the graphical behavior of MTSF, availability and profit of the system model.

\section{NOTATIONS}

$M_{0}$ : Main unit operating in full capacity

$M_{r}$ : Main unit operating in reduced capacity

$M_{P m}$ : Main unit under preventive maintenance

$M_{F}:$ Failure of main unit

$R_{o}:$ Reserve unit in operation

$R_{S}:$ Reserve unit in standby

$R_{P m}:$ Reserve unit under preventive maintenance

$R_{F}:$ Failure of reserve unit

$\beta$ : Constant rate of taking the unit into preventive maintenance

$\beta_{1}$ : Constant rate of the main unit going into reduced capacity while reserved unit is in standby

$\beta_{2}$ : Constant rate of the main unit going into reduced capacity while reserved unit is in operation 
$\beta_{3}$ : Failure rate of the reserved unit in standby

$\beta_{4}$ : Failure rate of the main unit

$\beta_{5}$ : Failure of reserved unit in operation

$\alpha$ : Constant rate end of preventive maintenance

$\alpha_{1}$ : Minor maintenance rate of the main unit

$\alpha_{2}$ : Major maintenance rate of the main unit

$\alpha_{3}$ : repair rate of reserved unit in standby

$\alpha_{4}$ : repair rate of main unit

$\alpha_{5}$ : repair rate of the reserved unit in operation

\section{SYSTEM DESCRIPTION \\ ASSUMPTIONS}

AND

\subsection{Assumptions}

1. The system consists of two non identical units: Main and Reserve unit.

2. Reserve unit is a warm standby unit

3. The system is attended by one repairman

4. Repair of the failed unit has priority over minor and major maintenance.

5. System failed when the units have failed

6. In high temperature condition, the main unit will be in operational while the reserve unit will be on standby (warm)

7. As transition from high temperature condition to low temperature, the main will work in reduced capacity because of the usage while the reserve is either on standby or in operation depending upon the strength of the main unit

\subsection{States Of The System}

$S_{0}\left(M_{O}, R_{S}\right)$, $S_{1}\left(M_{r}, R_{S}\right)$

$S_{2}\left(M_{r}, R_{O}\right), S_{3}\left(M_{r}, R_{F}\right), S_{4}\left(M_{F}, R_{O}\right)$,

$S_{5}\left(M_{F}, R_{F}\right), S_{6}\left(M_{P m}, R_{P m}\right)$

Up States:

$$
\begin{aligned}
& S_{0}\left(M_{O}, R_{S}\right), S_{1}\left(M_{r}, R_{S}\right), S_{2}\left(M_{r}, R_{O}\right), \\
& S_{3}\left(M_{r}, R_{F}\right), S_{4}\left(M_{F}, R_{O}\right), S_{6}\left(M_{P m}, R_{P m}\right)
\end{aligned}
$$

Down State: $S_{5}\left(M_{F}, R_{F}\right)$

\section{MEAN TIME TO SYSTEM FAILURE}

From Fig. 1 let $P_{i}(t)$ be the probability that the system is in state $S_{i}$ at time $t \geq 0$. Let $P(t)$ be the probability row vector at time $t$. We have the following initial condition:

$$
\begin{aligned}
P(0) & =\left[P_{0}(0), P_{1}(0), P_{2}(0), P_{3}(0), P_{4}(0), P_{5}(0), P_{6}(0)\right] \\
& =[1,0,0,0,0,0,0]
\end{aligned}
$$

We obtain the following differential equations from Fig. 1.

$$
\begin{aligned}
& \frac{d P_{0}(t)}{d t}=-\left(\beta+\beta_{1}+\beta_{2}\right) P_{0}(t)+\alpha_{1} P_{1}(t)+\alpha_{2} P_{2}(t)+\alpha P_{6}(t) \\
& \frac{d P_{1}(t)}{d t}=-\left(\alpha_{1}+\beta_{3}\right) P_{1}(t)+\beta_{1} P_{0}(t)+\alpha_{3} P_{3}(t) \\
& \frac{d P_{2}(t)}{d t}=-\left(\alpha_{2}+\beta_{4}\right) P_{2}(t)+\beta_{2} P_{0}(t)+\alpha_{4} P_{4}(t) \\
& \frac{d P_{3}(t)}{d t}=-\left(\alpha_{3}+\beta_{4}\right) P_{3}(t)+\beta_{3} P_{1}(t)+\alpha_{4} P_{5}(t) \\
& \frac{d P_{4}(t)}{d t}=-\left(\alpha_{4}+\beta_{5}\right) P_{4}(t)+\beta_{4} P_{2}(t)+\alpha_{5} P_{5}(t) \\
& \frac{d P_{5}(t)}{d t}=-\left(\alpha_{4}+\alpha_{5}\right) P_{5}(t)+\beta_{4} P_{3}(t)+\beta_{5} P_{4}(t) \\
& \frac{d P_{6}(t)}{d t}=-\alpha P_{6}(t)+\beta P_{0}(t)
\end{aligned}
$$

The differential equations above can be transform to matrix

form as $\dot{P}=A_{1} P$

where

$\left.\begin{array}{ccc}0 & 0 & \alpha \\ 0 & 0 & 0 \\ \alpha_{4} & 0 & 0 \\ 0 & \alpha_{4} & 0 \\ -\left(\alpha_{4}+\beta_{5}\right) & \alpha_{5} & 0 \\ \beta_{5} & -\left(\alpha_{4}+\alpha_{5}\right) & 0 \\ 0 & 0 & -\alpha\end{array}\right]$

It is difficult to evaluate the transient solutions hence we delete the rows and columns of absorbing state of matrix $A_{1}$ and take the transpose to produce a new matrix, say $Q$. Following $[1,2]$ the expected time to reach an absorbing state is obtained from: 
$\operatorname{MTSF}=P(0)\left(-Q^{-1}\right)\left[\begin{array}{l}1 \\ 1 \\ 1 \\ 1 \\ 1 \\ 1\end{array}\right]=\frac{N_{1}}{D_{1}}$

$N_{1}=$

$\alpha\left(\alpha_{1} \alpha_{2} \alpha_{3} \alpha_{4}+\alpha_{1} \alpha_{3} \beta_{4} \beta_{5}+\alpha_{1} \alpha_{2} \alpha_{3} \beta_{5}+\alpha_{2} \alpha_{4} \beta_{3} \beta_{4}+\right.$

$\left.\alpha_{1} \alpha_{2} \alpha_{4} \beta_{4}+\alpha_{1} \alpha_{2} \beta_{4} \beta_{5}+\alpha_{1} \beta_{4}^{1} \beta_{5}+\beta_{3} \beta_{4}^{2} \beta_{5}+\alpha_{2} \beta_{3} \beta_{4} \beta_{5}\right)$

$\alpha \beta_{1}\left(\alpha_{2} \alpha_{3} \alpha_{4}+\alpha_{3} \beta_{4} \beta_{5}+\alpha_{2} \alpha_{3} \beta_{5}+\alpha_{2} \alpha_{4} \beta_{4}+\alpha_{2} \beta_{4} \beta_{5}+\beta_{4}\right.$ lie $\beta \beta_{5}$ ifferential equations in (1) above can be expressed as $\alpha \beta_{2}\left(\alpha_{1} \alpha_{3} \alpha_{4}+\alpha_{1} \alpha_{3} \beta_{5}+\alpha_{1} \alpha_{4} \beta_{4}+\alpha_{4} \beta_{3} \beta_{4}+\alpha_{1} \beta_{4} \beta_{5}+\right.$ $\left.\beta_{3} \beta_{4} \beta_{5}\right)+\alpha \beta_{1} \beta_{3}\left(\alpha_{2} \alpha_{4}+\beta_{4} \beta_{5}+\alpha_{2} \beta_{5}\right)+\alpha \beta\left(\alpha_{1} \alpha_{3}+\right.$ $\left.\alpha_{1} \beta_{4}+\beta_{3} \beta_{4}\right)+\beta\left(\alpha_{1} \alpha_{2} \alpha_{3} \alpha_{4}+\alpha_{1} \alpha_{3} \beta_{4} \beta_{5}+\alpha_{1} \alpha_{2} \alpha_{3} \beta_{5}+\right.$ $\alpha_{2} \alpha_{4} \beta_{3} \beta_{4}+\alpha_{1} \alpha_{2} \alpha_{4} \beta_{4}+\alpha_{1} \alpha_{2} \beta_{4} \beta_{5}+\alpha_{1} \beta_{4}^{2} \beta_{5}+\beta_{3} \beta_{4}^{2} \beta_{5}$ $+\alpha_{2} \beta_{3} \beta_{4} \beta_{5}$ )

$D_{1}=\alpha \beta\left(\alpha_{1} \alpha_{3} \beta_{2} \beta_{5}+\alpha_{2} \alpha_{4} \beta_{1} \beta_{3}+\alpha_{2} \beta_{1} \beta_{3} \beta_{5}+\beta_{1} \beta_{3} \beta_{4} \beta_{5}\right.$ $+\alpha_{1} \beta_{2} \beta_{4} \beta_{5}+\beta_{2} \beta_{3} \beta_{4} \beta_{5}$ )

where

$$
\left[\begin{array}{c}
\dot{P}_{0} \\
\dot{P}_{1} \\
\dot{P}_{2} \\
\dot{P}_{3} \\
\dot{P}_{4} \\
\dot{P}_{5} \\
\dot{P}_{6}
\end{array}\right]=\left[\begin{array}{ccccccc}
-\left(\beta+\beta_{1}+\beta_{2}\right) & \alpha_{1} & \alpha_{2} & 0 & 0 & 0 & \alpha \\
\beta_{1} & -\left(\alpha_{3}+\beta_{3}\right) & 0 & \alpha_{3} & 0 & 0 & 0 \\
\beta_{2} & 0 & -\left(\alpha_{2}+\beta_{4}\right) & 0 & \alpha_{4} & 0 & 0 \\
0 & \beta_{3} & 0 & -\left(\alpha_{3}+\beta_{4}\right) & 0 & \alpha_{4} & 0 \\
0 & 0 & \beta_{4} & 0 & -\left(\alpha_{4}+\beta_{5}\right) & \alpha_{5} & 0 \\
0 & 0 & 0 & \beta_{4} & \beta_{5} & -\left(\alpha_{4}+\alpha_{5}\right) & 0 \\
\beta & 0 & 0 & 0 & 0 & 0 & -\alpha
\end{array}\right]\left[\begin{array}{c}
P_{0} \\
P_{1} \\
P_{2} \\
P_{3} \\
P_{4} \\
P_{5} \\
P_{6}
\end{array}\right]
$$

\section{AVAILABILITY ANALYSIS}

\section{Using the same initial conditions}

$$
P(0)=\left[P_{0}(0), P_{1}(0), P_{2}(0), P_{3}(0), P_{4}(0), P_{5}(0), P_{6}(0)\right]
$$

The system availability can be obtained from the solutions for $P_{i}(t), i=0,1,2, \ldots, 6$. The states $0,1,2,3,4$ and 6 in

Fig. 1 are the only working states of the system.

The steady-state availability is given by

$A(\infty)=P_{0}(\infty)+P_{1}(\infty)+P_{2}(\infty)+P_{3}(\infty)+P_{4}(\infty)+P_{6}(\infty)$

In the steady state, the derivatives of the state probabilities become zero so that

$A_{1} P=0$

which in matrix 


$\left[\begin{array}{ccccccc}-\left(\beta+\beta_{1}+\beta_{2}\right) & \alpha_{1} & \alpha_{2} & 0 & 0 & 0 & \alpha \\ \beta_{1} & -\left(\alpha_{3}+\beta_{3}\right) & 0 & \alpha_{3} & 0 & 0 & 0 \\ \beta_{2} & 0 & -\left(\alpha_{2}+\beta_{4}\right) & 0 & \alpha_{4} & 0 & 0 \\ 0 & \beta_{3} & 0 & -\left(\alpha_{3}+\beta_{4}\right) & 0 & \alpha_{4} & 0 \\ 0 & 0 & \beta_{4} & 0 & -\left(\alpha_{4}+\beta_{5}\right) & \alpha_{5} & 0 \\ 0 & 0 & 0 & \beta_{4} & \beta_{5} & -\left(\alpha_{4}+\alpha_{5}\right) & 0 \\ \beta & 0 & 0 & 0 & 0 & 0 & -\alpha\end{array}\right]\left[\begin{array}{l}P_{0} \\ P_{1} \\ P_{2} \\ P_{3} \\ P_{4} \\ P_{5} \\ P_{6}\end{array}\right]=\left[\begin{array}{c}0 \\ 0 \\ 0 \\ 0 \\ 0 \\ 0 \\ 0\end{array}\right]$

Using the following normalizing condition:

$P_{0}(\infty)+P_{1}(\infty)+P_{2}(\infty)+P_{3}(\infty)+P_{4}(\infty)+P_{5}(\infty)+P_{6}(\infty)=1(5)$

Substituting (5) in (4) we have the following:

$$
\left[\begin{array}{ccccccc}
-\left(\beta+\beta_{1}+\beta_{2}\right) & \alpha_{1} & \alpha_{2} & 0 & 0 & 0 & \alpha \\
\beta_{1} & -\left(\alpha_{3}+\beta_{3}\right) & 0 & \alpha_{3} & 0 & 0 & 0 \\
\beta_{2} & 0 & -\left(\alpha_{2}+\beta_{4}\right) & 0 & \alpha_{4} & 0 & 0 \\
0 & \beta_{3} & 0 & -\left(\alpha_{3}+\beta_{4}\right) & 0 & \alpha_{4} & 0 \\
0 & 0 & \beta_{4} & 0 & -\left(\alpha_{4}+\beta_{5}\right) & \alpha_{5} & 0 \\
0 & 0 & 0 & \beta_{4} & \beta_{5} & -\left(\alpha_{4}+\alpha_{5}\right) & 0 \\
1 & 1 & 1 & 1 & 1 & 1 & 1
\end{array}\right]\left[\begin{array}{c}
P_{0} \\
P_{1} \\
P_{2} \\
P_{3} \\
P_{4} \\
P_{5} \\
P_{6}
\end{array}\right]=\left[\begin{array}{l}
0 \\
0 \\
0 \\
0 \\
0 \\
0 \\
1
\end{array}\right]
$$

We solve for the system linear of equations in the matrix above to obtain the steady-state probabilities

$P_{0}(\infty), P_{1}(\infty), P_{2}(\infty), P_{3}(\infty), P_{4}(\infty), P_{6}(\infty)$

The steady-state availability is given by :

$A(\infty)=\frac{N_{2}}{D_{2}}$

$N_{2}=\alpha \alpha_{4}\left(\alpha_{1} \alpha_{3} \beta_{4} \beta_{5}+\alpha_{1} \alpha_{2} \alpha_{3} \beta_{5}+\alpha_{1} \alpha_{2} \alpha_{3} \alpha_{4}+\alpha_{1} \alpha_{2} \alpha_{3} \alpha_{5}+\right.$

$\left.\alpha_{1} \alpha_{2} \beta_{4} \beta_{5}+\alpha_{2} \alpha_{5} \beta_{3} \beta_{4}\right)+\alpha \alpha_{4}\left(\alpha_{2} \alpha_{3} \alpha_{4} \beta_{1}+\alpha_{2} \alpha_{3} \alpha_{5} \beta_{1}+\right.$

$\left.\alpha_{2} \alpha_{3} \beta_{1} \beta_{5}+\alpha_{2} \alpha_{5} \beta_{1} \beta_{4}+\alpha_{3} \beta_{1} \beta_{4} \beta_{5}+\alpha_{3} \beta_{2} \beta_{4} \beta_{5}\right)+\alpha \alpha_{4}\left(\alpha_{1} \alpha_{3} \beta_{2} \beta_{5}\right.$

$+\alpha_{1} \alpha_{3} \alpha_{5} \beta_{2}+\alpha_{1} \alpha_{3} \alpha_{4} \beta_{2}+\alpha_{1} \alpha_{5} \beta_{2} \beta_{4}+\alpha_{5} \beta_{2} \beta_{3} \beta_{4}+$

$\left.\alpha_{5} \beta_{1} \beta_{3} \beta_{4}\right)+\alpha \alpha_{4}\left(\alpha_{1} \beta_{2} \beta_{4} \beta_{5}+\alpha_{2} \alpha_{4} \beta_{1} \beta_{3}+\alpha_{2} \alpha_{5} \beta_{1} \beta_{3}+\right.$

$\left.\alpha_{2} \beta_{1} \beta_{3} \beta_{5}+\beta_{1} \beta_{3} \beta_{4} \beta_{5}+\beta_{2} \beta_{3} \beta_{4} \beta_{5}\right)+\alpha \beta_{4}\left(\alpha_{1} \alpha_{5} \beta_{2} \beta_{4}+\right.$

$\left.\alpha_{1} \alpha_{3} \alpha_{4} \beta_{2}+\alpha_{1} \alpha_{3} \alpha_{5} \beta_{2}+\alpha_{2} \alpha_{5} \beta_{1} \beta_{3}+\alpha_{5} \beta_{1} \beta_{3} \beta_{4}+\alpha_{5} \beta_{2} \beta_{3} \beta_{4}\right)+$

$\alpha_{4} \beta\left(\alpha_{1} \alpha_{3} \beta_{4} \beta_{5}+\alpha_{1} \alpha_{2} \alpha_{3} \beta_{5}+\alpha_{1} \alpha_{2} \alpha_{3} \alpha_{4}+\alpha_{1} \alpha_{2} \alpha_{3} \alpha_{5}+\right.$

$\left.\alpha_{1} \alpha_{2} \alpha_{5} \beta_{4}+\alpha_{2} \alpha_{5} \beta_{3} \beta_{4}\right)$

$D_{2}=\alpha \beta_{1} \beta_{3} \beta_{4}^{2} \beta_{5}+\alpha \alpha_{2} \alpha_{4} \beta_{1} \beta_{3} \beta_{5}+\alpha \alpha_{2} \alpha_{4} \alpha_{5} \beta_{1} \beta_{3}+\alpha \alpha_{2} \alpha_{4}^{2} \beta_{1} \beta_{3}$

$+\alpha \beta_{2} \beta_{3} \beta_{4}^{2} \beta_{5}+\alpha \alpha_{5} \beta_{1} \beta_{3} \beta_{4}^{2}+\alpha \alpha_{5} \beta_{2} \beta_{3} \beta_{4}^{2}+$

$\alpha \alpha_{4} \beta_{1} \beta_{3} \beta_{4} \beta_{5}+\alpha \alpha_{2} \beta_{1} \beta_{3} \beta_{4} \beta_{5}+\alpha \alpha_{4} \beta_{2} \beta_{3} \beta_{4} \beta_{5}+\alpha \alpha_{2} \alpha_{4} \alpha_{5} \beta_{3} \beta_{4}+$

$\alpha \alpha_{2} \alpha_{4} \beta_{1} \beta_{3} \beta_{4}+\alpha \alpha_{4} \alpha_{5} \beta_{2} \beta_{3} \beta_{4}+\alpha_{2} \alpha_{4} \alpha_{5} \beta_{3} \beta_{4} \beta+$

$\alpha \alpha_{2} \alpha_{4} \alpha_{5} \beta_{1} \beta_{4}+\alpha \alpha_{4} \alpha_{5} \beta_{1} \beta_{3} \beta_{4}+\alpha \alpha_{2} \alpha_{5} \beta_{1} \beta_{3} \beta_{4}+\alpha \alpha_{3} \alpha_{4} \beta_{2} \beta_{4} \beta_{5}+$

$\alpha \alpha_{3} \alpha_{4} \beta_{1} \beta_{4} \beta_{5}+\alpha \alpha_{2} \alpha_{3} \alpha_{4} \beta_{1} \beta_{5}+\alpha \alpha_{2} \alpha_{3} \alpha_{4}^{2} \beta_{1}+$

$\alpha \alpha_{2} \alpha_{3} \alpha_{4} \alpha_{5} \beta_{1}+\alpha_{1} \alpha_{3} \alpha_{4} \beta_{4} \beta_{5} \beta+\alpha \alpha_{1} \alpha_{3} \alpha_{4} \beta_{4} \beta_{5}+\alpha \alpha_{1} \alpha_{3} \beta_{2} \beta_{4} \beta_{5}+$

$\alpha \alpha_{1} \alpha_{3} \alpha_{4} \beta_{2} \beta_{4}+\alpha \alpha_{1} \alpha_{3} \alpha_{5} \beta_{2} \beta_{4}+\alpha \alpha_{1} \alpha_{3} \alpha_{4} \beta_{2} \beta_{5}+$ 
$\alpha \alpha \alpha_{3} \alpha_{4} \beta_{5} \beta+\alpha \alpha_{1} \alpha_{2} \alpha_{3} \alpha_{4} \beta_{5}+\alpha_{1} \alpha_{2} \alpha_{3} \alpha_{4}^{2} \beta+\alpha \alpha_{1} \alpha_{3} \alpha_{4}^{2} \beta_{2}+$

$\alpha_{1} \alpha_{2} \alpha_{3} \alpha_{4} \alpha_{5} \beta+\alpha \alpha_{1} \alpha_{3} \alpha_{4} \alpha_{5} \beta_{2}+\alpha \alpha_{1} \alpha_{2} \alpha_{3} \alpha_{4}^{2}+$

$\alpha \alpha_{1} \alpha_{2} \alpha_{3} \alpha_{4} \alpha_{5}+\alpha \alpha_{1} \beta_{2} \beta_{4}^{2} \beta_{5}+\alpha \alpha_{1} \alpha_{5} \beta_{2} \beta_{4}^{2}+\alpha \alpha_{1} \alpha_{4} \beta_{2} \beta_{4} \beta_{5}+$

$\alpha \alpha_{1} \alpha_{2} \alpha_{4} \alpha_{5} \beta_{4}+\alpha_{1} \alpha_{2} \alpha_{4} \alpha_{5} \beta_{4} \beta+\alpha \alpha_{1} \alpha_{4} \alpha_{5} \beta_{2} \beta_{4}$

\section{BUSY PERIOD ANALYSIS OF REPAIRMAN}

Using the same initial conditions as for the reliability case:

$$
\begin{aligned}
P(0) & =\left[P_{0}(0), P_{1}(0), P_{2}(0), P_{3}(0), P_{4}(0), P_{5}(0), P_{6}(0)\right] \\
& =[1,0,0,0,0,0,0]
\end{aligned}
$$

Let $B(\infty)$ be the probability that the repairman is busy in conducting corrective maintenance. In the steady state, the derivatives of the state probabilities become zero this will enable us to compute steady state busy:

$$
\begin{aligned}
& B(\infty)=P_{3}(\infty)+P_{4}(\infty)+P_{5}(\infty)=\frac{N_{3}}{D_{2}} \\
& N_{3}=\alpha \alpha_{4}\left(\alpha_{1} \beta_{2} \beta_{2} \beta_{5}+\alpha_{2} \alpha_{4} \beta_{1} \beta_{3}+\alpha_{2} \alpha_{5} \beta_{1} \beta_{3}+\alpha_{2} \beta_{1} \beta_{3} \beta_{5}+\right. \\
& \left.\beta_{1} \beta_{3} \beta_{4} \beta_{5}+\beta_{2} \beta_{3} \beta_{4} \beta_{5}\right)+\alpha \beta_{4}\left(\alpha_{1} \alpha_{5} \beta_{2} \beta_{4}+\alpha_{1} \alpha_{3} \alpha_{4} \beta_{2}+\right. \\
& \left.\alpha_{1} \alpha_{3} \alpha_{5} \beta_{2}+\alpha_{2} \alpha_{5} \beta_{1} \beta_{3}+\alpha_{5} \beta_{1} \beta_{3} \beta_{4}+\alpha_{5} \beta_{2} \beta_{3} \beta_{4}\right)+\alpha \beta_{4}\left(\alpha_{1} \alpha_{3} \beta_{2} \beta_{5}+\right. \\
& \alpha_{1} \beta_{2} \beta_{4} \beta_{5}+\alpha_{2} \alpha_{4} \beta_{1} \beta_{3}+\alpha_{2} \beta_{1} \beta_{3} \beta_{5}+\beta_{2} \beta_{3} \beta_{4} \beta_{5}+\beta_{1} \beta_{3} \beta_{4} \beta_{5}
\end{aligned}
$$

\section{EXPECTED FREQUECY OF MINOR MAINTENANCE}

Using the same initial conditions as for the reliability case:

$$
\begin{aligned}
P(0) & =\left[P_{0}(0), P_{1}(0), P_{2}(0), P_{3}(0), P_{4}(0), P_{5}(0), P_{6}(0)\right] \\
& =[1,0,0,0,0,0,0]
\end{aligned}
$$

Let $M(\infty)$ be the probability that the repairman is busy in conducting minor

Maintenance to the main unit working in reduced capacity

In the steady state, the derivatives of the state probabilities become

zero this will enable us to compute steady state minor maintenance:

$$
\begin{aligned}
& M(\infty)=P_{1}(\infty)=\frac{N_{4}}{D_{2}} \\
& N_{4}=\alpha \alpha_{4}\left(\alpha_{2} \alpha_{3} \alpha_{4} \beta_{1}+\alpha_{2} \alpha_{3} \alpha_{5} \beta_{1}+\alpha_{2} \alpha_{3} \beta_{1} \beta_{5}+\right. \\
& \left.\alpha_{2} \alpha_{5} \beta_{1} \beta_{4}+\alpha_{3} \beta_{1} \beta_{4} \beta_{5}+\alpha_{3} \beta_{2} \beta_{4} \beta_{5}\right)
\end{aligned}
$$

\section{EXPECTED FREQUENCY OF MAJOR MAINTENNCE}

Using the same initial conditions as for the reliability case:

$$
\begin{aligned}
P(0) & =\left[P_{0}(0), P_{1}(0), P_{2}(0), P_{3}(0), P_{4}(0), P_{5}(0), P_{6}(0)\right] \\
& =[1,0,0,0,0,0,0]
\end{aligned}
$$

In the steady state, the derivatives of the state probabilities become zero this will enable us to compute steady state major maintenance:

$$
\begin{aligned}
& K(\infty)=P_{2}(\infty)=\frac{N_{5}}{D_{2}} \\
& N_{5}=\alpha \alpha_{4}\left(\alpha_{1} \alpha_{3} \beta_{2} \beta_{5}+\alpha_{1} \alpha_{3} \alpha_{5} \beta_{2}+\alpha_{1} \alpha_{3} \alpha_{4} \beta_{2}+\right. \\
& \left.\alpha_{1} \alpha_{5} \beta_{2} \beta_{4}+\alpha_{5} \beta_{2} \beta_{3} \beta_{4}+\alpha_{5} \beta_{1} \beta_{3} \beta_{4}\right)
\end{aligned}
$$




\section{EXPECTED FREQUENCY OF PREVENTIVE MAINTENANCE}

Following $[1,2,3]$, in the steady state the derivatives of state probabilities become zero so that enables us to compute $P_{6}(\infty)$. We solve the system of linear equations in using normalizing condition in (3). The expected frequency of preventive maintenance is:

$$
\begin{aligned}
& P F(\infty)=P_{6}(\infty)=\frac{N_{6}}{D_{2}} \\
& N_{6}=\alpha_{4} \beta\left(\alpha_{1} \alpha_{3} \beta_{4} \beta_{5}+\alpha_{1} \alpha_{2} \alpha_{3} \beta_{5}+\alpha_{1} \alpha_{2} \alpha_{3} \alpha_{4}+\right. \\
& \left.\alpha_{1} \alpha_{2} \alpha_{3} \alpha_{5}+\alpha_{1} \alpha_{2} \alpha_{5} \beta_{4}+\alpha_{2} \alpha_{5} \beta_{3} \beta_{4}\right)
\end{aligned}
$$

\section{PROFIT ANALYSIS}

The units are subjected to corrective maintenance and preventive maintenance as can be observed in state 1,2,3,4 and 6from Fig. 1 the repairman performed

preventive maintenance action to the units before failure in state 7 and performed corrective maintenance to failed units in state 2,3,4,5,6. Let $C_{0}, C_{1}, C_{2}, C_{3}$ and $C_{4}$ be the revenue generated when the system is in working state and no income when in failed state, cost of each repair (corrective maintenance), minor maintenance, major maintenance and overhaul (preventive maintenance)

$P F=C_{0} A V(\infty)-C_{1} B(\infty)-C_{2} M(\infty)-C_{3} K(\infty)-C_{4} F(\infty)$

(10)

\subsection{PARTICULAR EXAMPLE}

\section{Case I:}

$\alpha_{1}=0.7$

$$
\alpha_{2}=\frac{1}{2} \alpha_{1}
$$

$\alpha_{3}=0.4, \alpha_{4}=0.7, \alpha_{5}=1.8$,

$\beta_{1}=2, \beta_{2}=0.2, \beta_{3}=0.9, \beta_{5}=1.5, \alpha=\beta$,

$C_{0}=4, C_{1}=3, C_{2}=2.5, C_{3}=2.7, C_{4}=2.2$,

And vary $\beta_{4}$ we plots Fig. 2 - 4

Case II:

$$
\begin{aligned}
& \alpha_{1}=0.7, \alpha_{2}=\frac{1}{2} \alpha_{1}, \alpha_{4}=0.7, \alpha_{5}=1.8 \beta_{1}=2, \\
& \beta_{2}=0.2, \beta_{3}=0.9, \beta_{4}=0.7, \beta_{5}=1.5, \alpha=\beta, \\
& C_{0}=4, C_{1}=3, C_{2}=2.5, C_{3}=2.7, C_{4}=2.2, \\
& \quad \text { and vary } \alpha_{4} \text { we plots Fig. } 5-7
\end{aligned}
$$

\section{CONCLUSION}

In this study, we developed the explicit expressions for MTSF, system availability, busy period, frequency of minor, major, preventive maintenance and profit function for the system. For the study of system behavior, we plot graphs in Fig. 2 - 4 for MTSF, system availability and profit function with respect to $\beta_{4}$ and Fig. 5 - 7 with respect to $\alpha_{4}$. From Fig. $2-4$, it is clear that MTSF, availability and profit decreases with increase in the value of $\beta_{4}$. Similarly, from Fig. $5-7$ it is clear that MTSF, availability and profit increases with increase in the value of $\alpha_{4}$.From the simulations, it is clear that measures of effectiveness like MTSF, availability and profit increases more with respect to $\alpha_{4}$. Hence it is vital to give preference to main unit over reserve unit in terms of repair and other maintenance action

\section{REFERENCES}

[1] El-Said, K.M.,. ( 2008).Cost analysis of a system with preventive maintenance by using Kolmogorov's forward equations method. Ame. J. of App. Sci. 5(4), 405-410

[2 ] El Sherbeny, M.S. (2012). Optimal system for warm standby components in the presence of standby switching failures, two types of failures and general repair time. IJCA, Vol. 44, no. 20, pp 17-26

[3] El Damcese, M.A. and Temraz, N.S. (2011). Availability and reliability measures for multi-state system by using Markov reward model. RT\&A, Vol. 2, No.3, 68-85

[4] Frenkel, I., Lisnianski, A. and Khvatskin, L. (2009). Corrective and reliability associated cost estimation of aging multi-state systems. Computer modeling and new technolonogy, Vol. 13, No. 1, 32-38

[5] Haggag, M.Y., (2009). Cost analysis of a system involving common cause failures and preventive maintenance.J. Maths. And Stat. 5(4), 305-310

[6] Kumar, A. and Malik, S.C. (2012). Stochastic Modeling of a computer system with priority to Preventive maintenance over software replacement subject to maximum operation and repair times, IJCA, Vol.43, no. $3,27-34$

[7] Wang, K., Yen, T and Fang, Y. (2012). Comparison of availability between two systems with warm standby units and different imperfect coverage. Quality technology and quantitative management, Vol. 9, No. 3, 265-282

[8] Wang, K.., Hseih, C. and Liou, C. (2006). Cost benefit analysis of series systems with cold standby components and a repairable service station. Quality technology and quantitative management, Vol. 3, No. 1, 77-9 


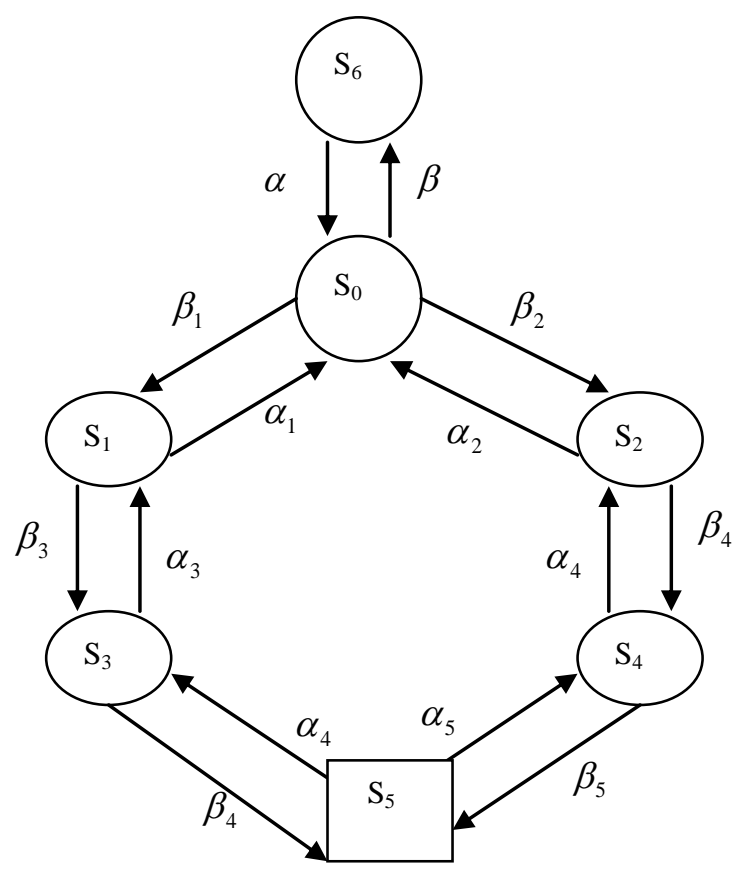

Fig. 1 schematic diagram of the Model

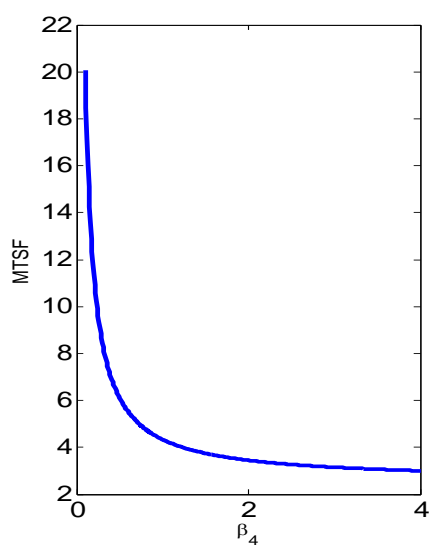

Fig.2 MTSF vs $\beta_{4}$

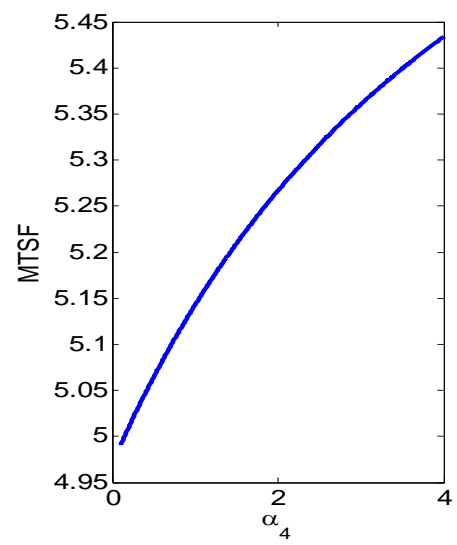

Fig.5 MTSF vs $\alpha_{4}$

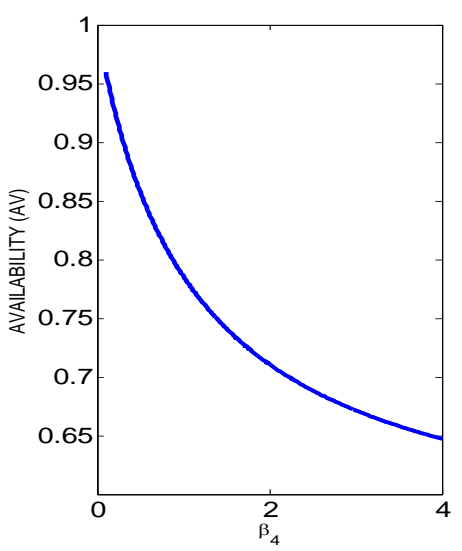

Fig. 3 Availability vs $\beta_{4}$

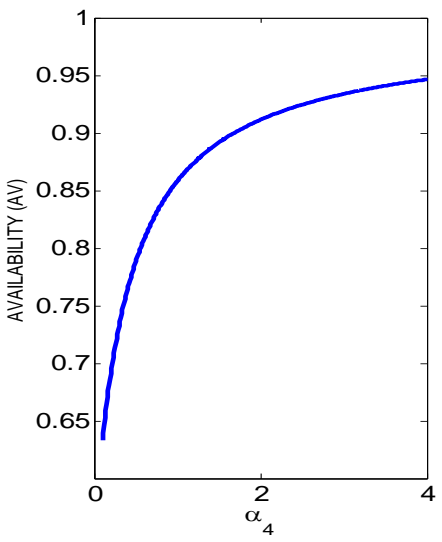

Fig. 6 Availability vs $\alpha_{4}$

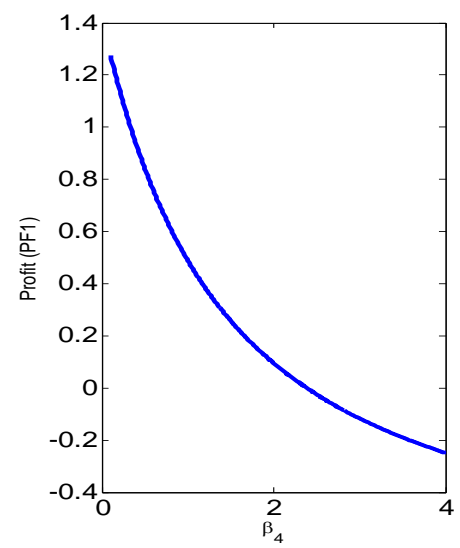

Fig. 4 Profit vs $\beta_{4}$

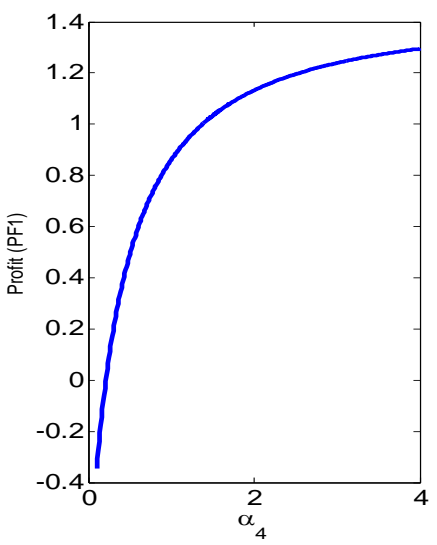

Fig. 7 Profit vs $\alpha_{4}$ 